\title{
Differences between the perspectives of physicians and patients on the potential barriers to optimal diabetes control in China: a multicenter study
}

Chun $\mathrm{Liu}^{1+}$, Shaoyong $\mathrm{Xu}^{1 \dagger}$, Jie Ming ${ }^{1 \dagger}$, Aihua Jia ${ }^{1,2}$, Yingji Wei $^{3}, \mathrm{Hui} \mathrm{Li}^{4}$, Yang Jiao ${ }^{5}$, Mingxi Song ${ }^{6}$, Yadong Zhao Yafang Du', Wenjuan Yang ${ }^{9}$, Xiaoqiang Lu ${ }^{10}$, Shengqi Shi ${ }^{11}$, Hui Tong ${ }^{2}$, Guangtang Jia ${ }^{12}$, Guohua Zhao ${ }^{13}$, Li Wang $^{14}$, Mei Zhang ${ }^{15}$, Junlin Wang ${ }^{16}$, Wenshu Liu ${ }^{17}$, Lin Fang ${ }^{18}$, Fuhong Dong ${ }^{19}$ and Qiuhe Ji ${ }^{1 *}$ (D)

\begin{abstract}
Background: To investigate the potential barriers to optimal diabetes control by evaluating the different perspectives of physicians and patients on such matters in China.

Methods: This multi-center survey was conducted from December 2015 to March 2016. A multi-stage stratified random sampling method was used to sample representative diabetes physicians and patients in 18 hospitals in Shaanxi province, China. A self-designed questionnaire was used. The questionnaire mainly consisted of 2 questions for physicians and 1 question for patients of which the participants were required to rank in priority of 3 (for physicians) and 2 (for patients) choices from a list of barriers. The strategies to improve diabetes control were only in the questionnaire for physicians.
\end{abstract}

Results: A total of 85 physicians and 584 patients completed the questionnaire. Physicians and patients differed regarding the patients' awareness of the risk of diabetes: over $70 \%$ of the physicians believed that the patients had no sufficient understanding of the harm and risk of diabetes, whereas the patients believed otherwise. Both physicians and patients considered self-monitoring of blood glucose to be an important link of glucose control; unfortunately, most of the patients failed to do so in practice. In addition, physicians considered "improving health insurance coverage for diabetes" as the first important measure and "providing more and easy-to-use diabetes brochures or educational materials for patients" as the second important measure to improve diabetes control.

Conclusion: The survey revealed differences between the perspectives of physicians and patients on the potential barriers to optimal diabetes control. The main potential barriers to optimal diabetes control were patient's poor lifestyle interventions, limited understanding of the danger of diabetes, and poor self-monitoring of blood glucose. From the physicians' perspective, China's primary focus about diabetes control in the future should still be put on diabetes education, particular the importance of lifestyle interventions.

Keywords: Diabetes control, Barriers, Cross-sectional study, China

\footnotetext{
* Correspondence: qiuheji@hotmail.com

${ }^{\dagger}$ Chun Liu, Shaoyong Xu and Jie Ming contributed equally to this work.

'Department of Endocrinology, Xijing Hospital, Air Force Medical University,

169 Changle Road West, Xi'an 710032, China

Full list of author information is available at the end of the article
}

(c) The Author(s). 2018 Open Access This article is distributed under the terms of the Creative Commons Attribution 4.0 International License (http://creativecommons.org/licenses/by/4.0/), which permits unrestricted use, distribution, and reproduction in any medium, provided you give appropriate credit to the original author(s) and the source, provide a link to the Creative Commons license, and indicate if changes were made. The Creative Commons Public Domain Dedication waiver (http://creativecommons.org/publicdomain/zero/1.0/) applies to the data made available in this article, unless otherwise stated. 


\section{Background}

Diabetes prevalence has been increasing dramatically in China in recent years. In 2010, the prevalence rate was $11.6 \%$ for diabetes and $50.1 \%$ for pre-diabetes in adults over 18 in China; the crude prevalence rate was $9.5 \%$ for diabetes and $35.5 \%$ for pre-diabetes in Shaanxi province; currently, the diabetic patient population in China accounts for approximately one-fourth of the diabetic patients worldwide [1]. With the rise in the overall incidence of diabetes and the incidence of diabetes in younger populations in China, individuals and the society are facing a higher burden of diabetes treatment. Unfortunately, even with continued innovation in anti-diabetic drugs and constantly evolving ideas regarding diabetic treatment, a substantial increase in expenses for diabetes care has failed to significantly improve the diabetes control rate; conversely, the morbidity of diabetes has increased each year [2]. Therefore, it is important to investigate the potential barriers to optimal diabetes control.

Diabetes physicians have gained rich experience in the daily diagnosis and treatment of diabetes, and therefore, their feedback on the potential barriers to optimal diabetes control is extremely valuable. Numerous Chinese and foreign studies have shown that from the perspective of physicians, the patient's lifestyle [3, 4], mental health [5], economic status [6], and religion [7] have a significant effect on diabetes control; furthermore, the physician's vocational education [8], specialist nurses [9], and education [10] also play important roles in diabetes control. However, the opinions differ between physicians in different surveys [3-6]. Notably, few such studies in the past have investigated the potential barriers to optimal diabetes control from the perspective of patients; consequently, an understanding of different perspectives between physicians and patients will, undoubtedly, provide an important reference for improving diabetes control and patient compliance in the future.

As such, this multi-center study was designed to investigate the potential barriers to optimal diabetes control by evaluating the different perspectives of physicians and patients on such matters, and also investigate the physician-recommended public health measures for improving diabetes control in China.

\section{Methods}

\section{Study design}

This multi-center cross-sectional survey was conducted from December 2015 to March 2016. A multi-stage stratified random sampling method was used to sample representative diabetes physicians and patients in Shaanxi province, China for this survey (Fig. 1).

Step 1 involved sampling physicians and patients in the cities. In addition to the capital city (Xi'an), physicians and patients from an economically developed prefecture- level city (Yulin, annual Gross National Products GDP > 50,000 yuan per capita), a moderately developed prefecture-level city (Baoji, annual GDP 30,000-50,000 yuan per capita), and a developing prefecture-level city (Ankang, annual GDP < 30,000 yuan per capita) were sampled; the GDP was classified according to the 2015 GDP in Shaanxi Province. This step was non-randomized. Step 2 involved stratifying the hospitals in the capital city and the three prefecture-level cities. The hospitals in the capital city were stratified into medical university hospitals, tertiary municipal hospitals, and secondary county hospitals; the hospitals in the prefecture-level cities were stratified into tertiary municipal hospitals and secondary county hospitals. Step 3 involved sampling the hospitals. Given the differences in the city sizes and the distribution of medical resources between the capital city and the prefecture-level cities, we used a simple random sampling (drawing) method to randomly sample three medical university hospitals, three tertiary municipal hospitals, and three secondary county hospitals in the capital city in addition to one tertiary municipal hospital and two secondary county hospitals in each of the prefecture-level cities. Thus, we sampled a total of 18 hospitals, including three medical university hospitals, six tertiary municipal hospitals, and nine secondary county hospitals. Step 4 involved sampling the physicians. According to the distribution of physicians in the different levels of the hospitals, we randomly sampled six residents, three attending physicians, and one chief physician from each medical university hospital; three residents, two attending physicians, and one chief physician from each tertiary municipal hospital; and two residents and one attending or chief physician from each secondary county hospital. Thus, we sampled a total of 93 physicians, including 54 residents, 21 to 31 attending physicians, and nine to 19 chief physicians. Step 5 involved sampling the patients. We used the cluster random sampling method and enrolled all of the patients with type 2 diabetes (based on World Health Organization 1999 criteria) who were hospitalized for diabetes treatment in department of endocrine during a certain time window and who met all of the study criteria, had poor diabetes control (HbA1c $\geq 7 \%$ ), and were willing to participate in this study, until the planned number of participants was reached. Given the difference in ward capacity between the different levels of hospitals, we planned to sample 40 patients in each medical university hospital and tertiary municipal hospital and 30 patients in each secondary county hospital, for a total of 630 patients, which provided a sufficient sample according to previous literatures [11-23].

\section{Data collection}

We developed our own questionnaire for data collection. While designing the questionnaire, we referred all the 


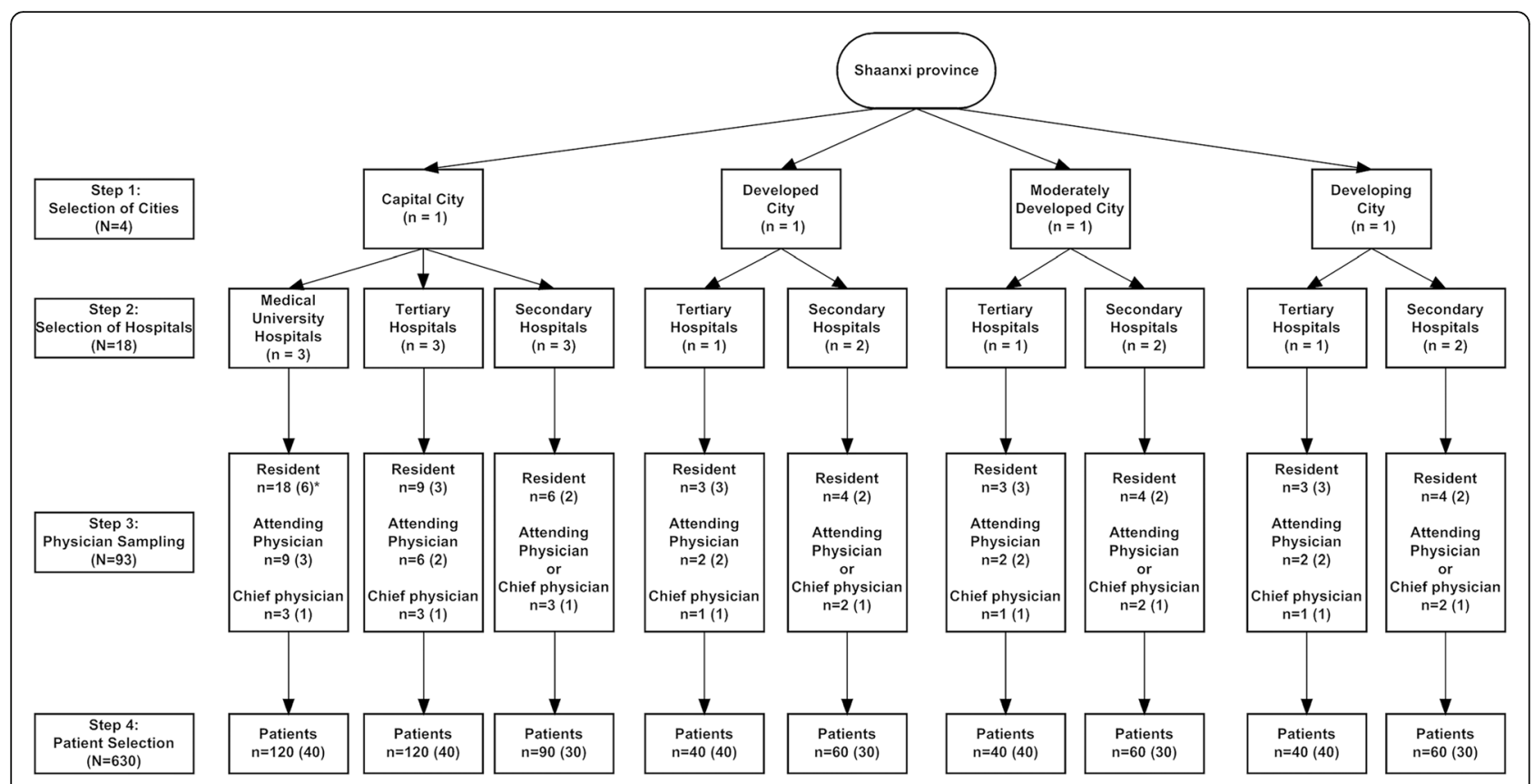

Fig. 1 Protocol design flowchart. *: numbers in each hospital

barriers and strategies from previous related literatures, and expanded some potential ones localized for the Chinese, which were from a small-scale pre-survey [11-23]. Two versions of the survey were used: the physician version and the patient version. (Additional file 1).

The physician questionnaire had two parts. Part 1 was used to collect basic information on the physician, including title and the type and location of the hospital. Part 2 contained two questions. Question 1 asked "According to your own clinical experience, what do you think are the top three potential barriers to optimal diabetes control?" There were three items could be chosen from a list of eight items. The physicians were asked to rank the factors in the order of importance. Question 2 asked "From the perspective of the government/community/hospital/physician, what do you think are the top three areas that require urgent improvement in order to improve diabetes control?" There were three items could be chosen from a list of nine items. Again, the physicians were asked to rank the areas in the order of importance.

The patient questionnaire contained two parts. Part 1 was used to collect patient demographic data, lifestyle, and history of diabetes. Part 2 contained only one question: "What do you (the patient) think are the causes of your uncontrolled blood glucose?" This was a multi-choice question and the patients could choose more than one factors for uncontrolled glucose. The patients were asked to mark the most important item. See the annex for the questionnaire design and baseline information.

\section{Statistical analysis}

EpiData software (version 2.3) was used to enter the data obtained into the database, and the Statistical Package for Social Sciences 20.0 (SPSS Inc., Chicago, IL, USA) was used for the descriptive statistics. The measurement data were expressed as the numerical mean and standard deviation. Count data are expressed as percentages (\%).

\section{Results}

\section{Physicians questionnaire}

A total of 93 physicians were sampled and asked to participate, of whom 85 agreed to participate and complete the physician questionnaire (response rate: 91.4\%), including $42(49.4 \%)$ residents, 31 (36.5\%) attending physicians, and 12 (14.1\%) chief physicians; 30 (35.3\%) physicians were from medical university hospitals, 35 (41.2\%) were from tertiary municipal (non-teaching) hospitals, and $20(23.5 \%)$ were from secondary county hospitals; 45 (52.9\%) physicians were from the capital city, 24 (28.2\%) were from prefecture-level cities, and 16 (18.8\%) were from counties or lower level cities (Table 1).

For question 1 (Figs. 2), "According to your own clinical experience, what do you think are the top three potential barriers to optimal diabetes control?", $62(62 / 85$ 72.9\%) physicians considered patient's "insufficient understanding of the danger of diabetes" as an important factor, of whom 33 (33/85 38.8\%) physicians ranked it as the most important factor, 23 (23/85 27.1\%) physicians ranked it as the second most important factor, and 6 (6/85 7.1\%) physicians ranked it as the third most important factor. 
Table 1 Baseline information of the physicians

\begin{tabular}{ll}
\hline Variable & $N=85$ \\
\hline Title, $\mathrm{n}(\%)$ & $42(49.4)$ \\
Resident & $31(36.5)$ \\
Attending Physician & $12(14.1)$ \\
Chief Physician & \\
Hospital, $\mathrm{n}(\%)$ & $30(35.3)$ \\
Medical university hospital & $35(41.2)$ \\
Tertiary non-teaching hospital & $20(23.5)$ \\
Secondary hospital & \\
Location, $\mathrm{n}(\%)$ & $45(52.9)$ \\
Capital city & $24(28.2)$ \\
Prefecture-level city & $16(18.8)$ \\
County and below &
\end{tabular}

In addition, 56 (56/85 65.9\%) physicians considered patient's "lack of perseverance to stick to lifestyle intervention (if even understanding the danger of diabetes)" as an important factor, of whom 29 (29/85 $34.1 \%)$ physicians ranked it as the most important factor, $21(21 / 8524.7 \%)$ physicians ranked it as the second most important factor, and 6 (6/85 7.1\%) physicians ranked it as the third most important factor.

Moreover, the physicians considered patient's failure to monitor blood glucose regularly, poor medication compliance, economic reasons, and the uneducated use of herbal medicine or health products as moderately effective potential barriers to optimal diabetes control. Whereas physicians considered factors that failure to attend scheduled follow-ups or inconvenience to purchase drugs as lower effective factors to diabetes control than other factors.
For question 2 (Figs. 3), "From the perspective of the government/community/hospital/physician, what do you think are the top three areas that require urgent improvement in order to improve diabetes control?", 38 (38/85 $44.7 \%$ ) physicians considered "improving health insurance coverage for diabetes" as an important measure, of whom 20 (20/85 23.5\%) physicians ranked it as the most important factor, $12(12 / 8514.1 \%)$ physicians ranked it as the second most important factor, and 6 (6/85 7.1\%) physicians ranked it as the third most important factor.

In addition, 33 (33/85 38.8\%) physicians considered "providing more and easy-to-use diabetes brochures or educational materials for patients" as an important factor, of whom $14(14 / 8516.5 \%)$ physicians ranked it as the most important factor, $12(12 / 8514.1 \%)$ physicians ranked it as the second most important factor, and 7 (7/ $858.2 \%)$ physicians ranked it as the third most important factor.

Moreover, although the physicians considered regulating media campaigns and minimizing false advertising, in addition to engaging or encouraging family members to become involved in the care of diabetic patients as important measures for strengthening public health management of diabetes, few ranked these factors as the most important factor. Furthermore, the physicians did not consider factors such as strengthening the training of diabetes specialists, training more specialist diabetes nurses to provide guidance for patients, and improving multidisciplinary and multi-specialty collaboration for diabetes control as urgent public health measures for improving diabetes control.

\section{Patient questionnaire}

A total of 630 patients were sampled and asked to participate, of whom 584 patients agreed to participate and

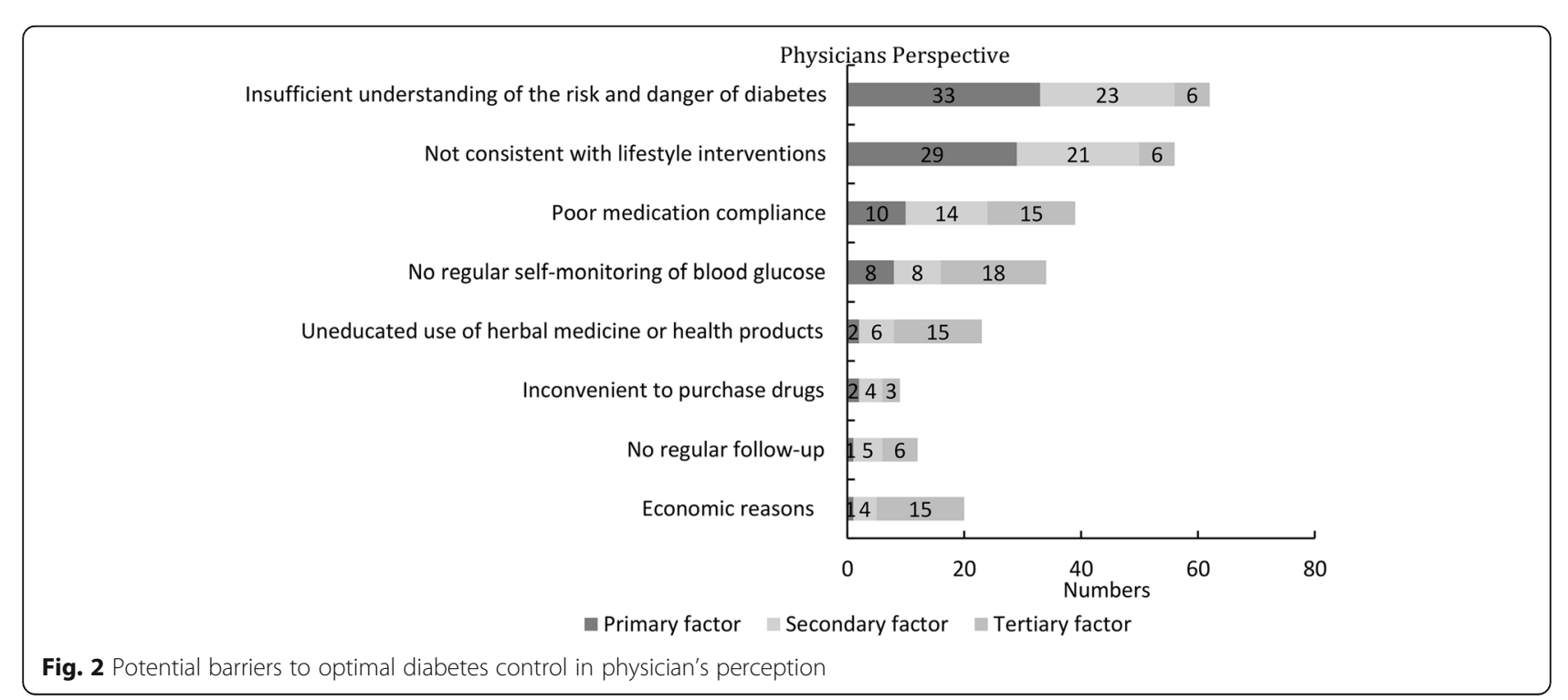




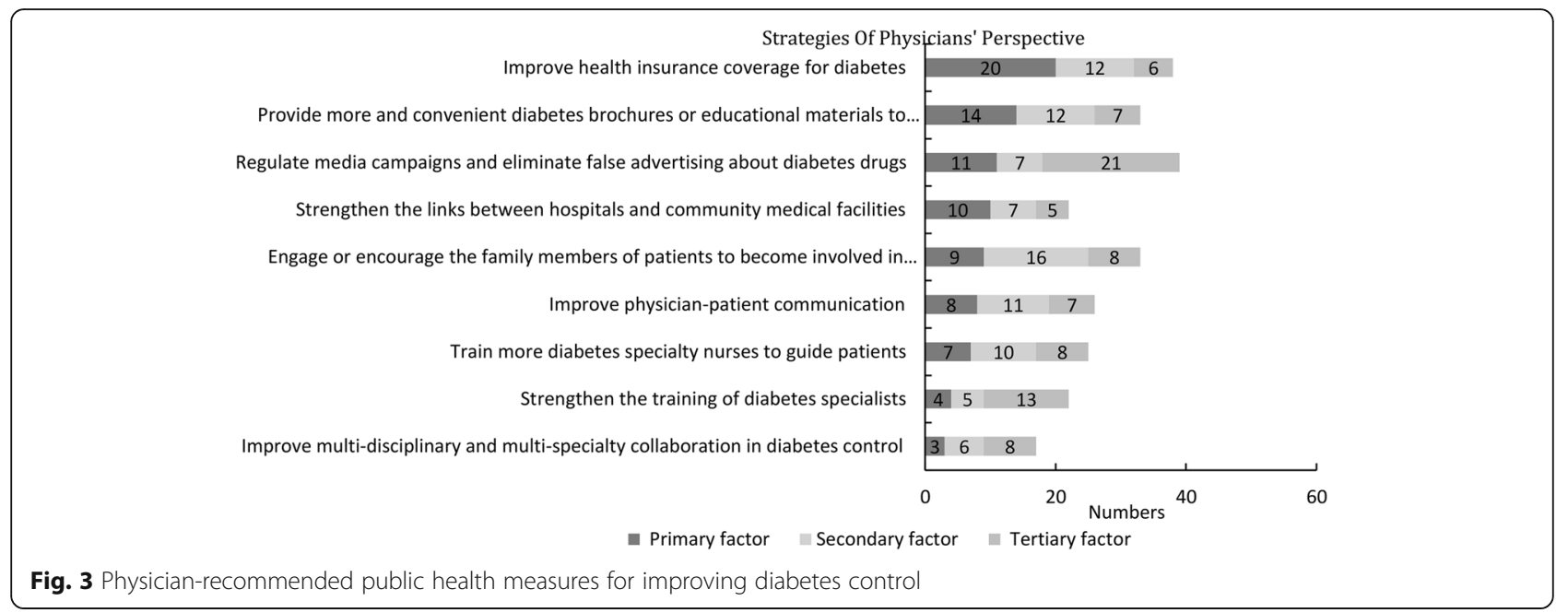

completed the patient questionnaire (response rate: $92.70 \%)$. Of the patients who completed the questionnaire, 325 patients were men with a mean age of 56.27 years $( \pm 13.19)$, and 254 patients were women with a mean age of 58.06 years ( \pm 12.79). Moreover, 242 (44.2\%) patients had been diagnosed with diabetes for less than 5 years, 175 (32.0\%) patients had been diagnosed with diabetes for 5 to 10 years, and 130 (23.8\%) patients had been diagnosed with diabetes for $\geq 10$ years (Table 2).

Table 2 Baseline information of the patients

\begin{tabular}{ll}
\hline Variable & $N=584$ \\
\hline Gender, n (\%) & $325(56.0)$ \\
Male & $254(43.8)$ \\
Female & $57.01 \pm 13.27$ \\
Mean age, years & $56.27 \pm 13.19$ \\
Male & $58.06 \pm 12.79$ \\
Female & \\
Educational level, n (\%) & $165(31.3)$ \\
Primary school and below & $226(42.9)$ \\
Middle and high school & $132(25.0)$ \\
College and above & \\
Economic condition ${ }^{\mathrm{a}}, \mathrm{n}(\%)$ & $79(13.5)$ \\
Poverty and below & $266(45.5)$ \\
Subsistence level & $194(33.2)$ \\
Well-to-do and above & \\
Duration of diabetes, $\mathrm{n}(\%)$ & $242(44.2)$ \\
$<5$ years & $175(32.0)$ \\
5 to 10 years & $130(23.8)$ \\
\hline 10 years & \\
\hline
\end{tabular}

${ }^{a}$ Economic condition was categorized according to per capita annual net income of households: poverty and below < 5000 China Yuan (CNY), subsistence level $5000-80,000$ CNY, and well-to-do and above $>80,000$ CNY
The data analysis of the 584 valid patient questionnaires showed that for the question, "What do you (the patient) think are the potential barriers to optimal diabetes control?" (Figs. 4), 338 (338/584 57.9\%) patients considered "I do not follow a proper diet" as an important barrier, of whom 142 (142/584 24.3\%) patients ranked it as the most important reason and 196 (196/ 584 33.6\%) patients ranked it as the second most important barrier. A total of 263 (263/584 45.0\%) patients considered "I do not exercise as instructed" as an important reason, of whom $55(55 / 584$ 9.4\%) patients ranked it as the most important barrier and 208 (208/ 584 35.6\%) patients ranked it as the second most important barrier.

Moreover, the patients considered failure to monitor blood glucose as instructed as an important potential barrier to optimal diabetes control. However, the patients did not consider high drug cost, inconvenience to purchase drugs, the use of herbal medicine, and incorrect treatment protocol as potential barriers to optimal diabetes control.

\section{Discussion}

This multi-center cross-sectional study showed that both physicians and patients considered lifestyle as one of the most important potential barriers to optimal diabetes control. However, physicians and patients differed regarding the patients' awareness of the risk of diabetes: over $70 \%$ of the physicians believed that the patients had no sufficient understanding of the harm and risk of diabetes, whereas the patients believed otherwise. In addition, this study showed that both physicians and patients considered self-monitoring of blood glucose to be an important link of glucose control; unfortunately, most of the patients failed to do so in practice.

First, this survey showed that both physicians and patients considered lifestyle as one of the most important 


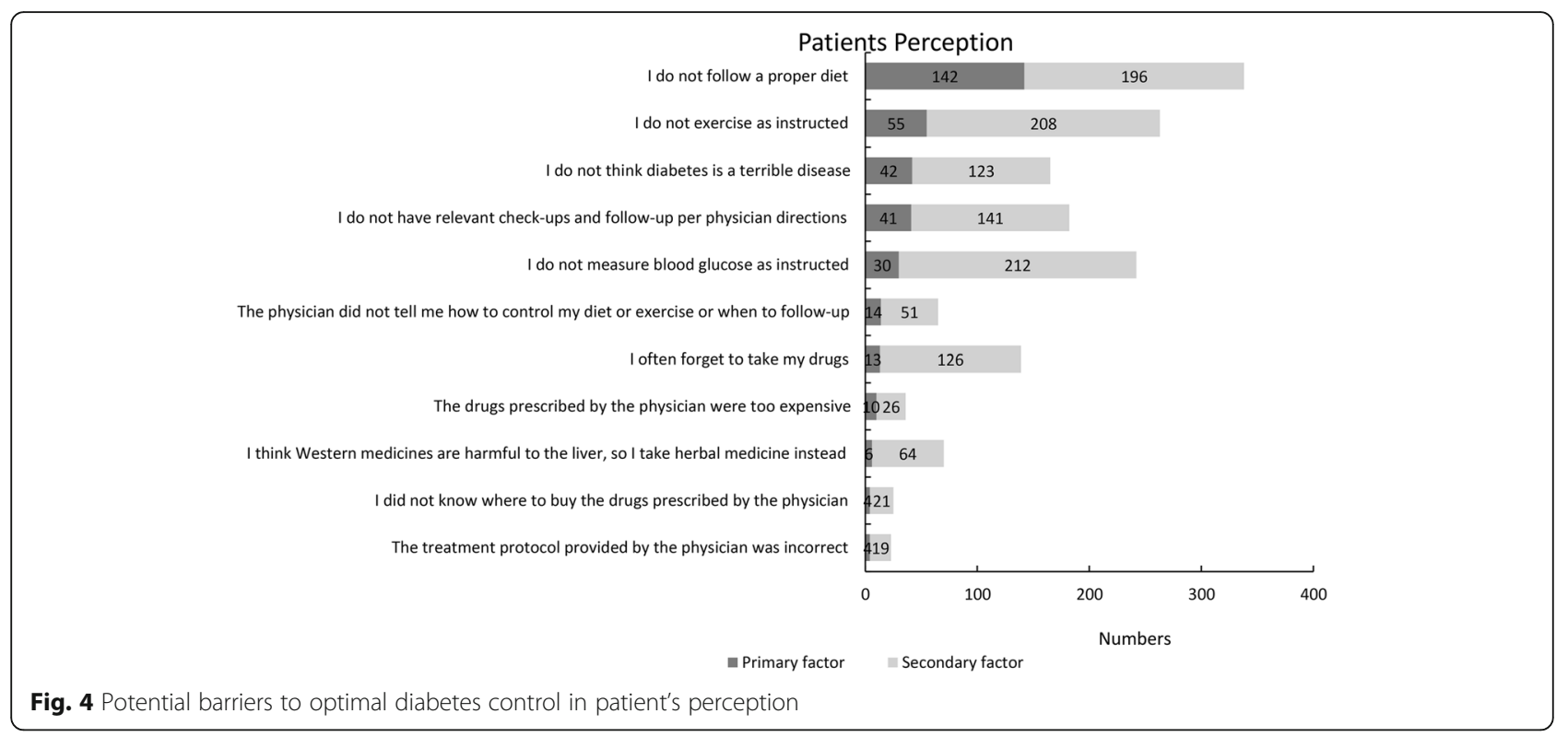

potential barriers to optimal diabetes control. "Lifestyle intervention" ranked second in the physician survey, but nearly $70 \%$ of the physicians considered it an important factor, suggesting that most physicians recognized its importance, which was consistent with survey results in The United States of America (US) [13], the Middle East, Thailand and South Africa [6, 15]. The patient survey showed that nearly $100 \%$ of the patients with poor diabetes control checked "I do not follow a proper diet" or "I do not exercise as instructed" or both, indicating that most Chinese diabetic patients recognized the importance of lifestyle intervention in diabetes control but did not stick to it [21], which is common in both China and other countries [12, 19]. Both physicians and patients considered lifestyle intervention an important factor, highlighting its importance in poor diabetes control in China. In terms of measures for this issue, physicians suggested "providing more and easy-to-use diabetes brochures or educational materials for patients" and "engaging or encouraging family members to become involved in the care of diabetic patients", which differed from the results in some developed countries. For example, some physicians in the US believed that comprehensive intervention was more important than providing more education materials [18] because in developed countries, patients already had access to basic health education and were generally well-informed. Innovation in drug research and development and the use of insulin could not substitute for the role of lifestyle interventions in improving diabetes control; conversely, a long-term poor lifestyle had adverse effects on drug and insulin therapy [20]. Therefore, we suggest that in Shaanxi Province, which displays uneven development, priority should be given to educational diabetes materials highlighting basic treatment. Meanwhile, active patient engagement [10] and effective self-management skills [14] are important for improving the disease control rate; thus, physicians should encourage and help the family members of patients become involved in diabetes monitoring and control.

Second, physicians and patients differed in the patients' awareness of the risk of diabetes. Over $70 \%$ of the physicians believed that patients had no sufficient understanding of the harm and risk of diabetes, which was consistent with the results in studies conducted in the Middle East, South Korea, and Japan in which physicians believed that patients should learn more about diabetes [16-18]. The importance of diabetes education has been established in the medical community [14, 21]. However, just under $30 \%$ of the patients considered "I do not think diabetes is a terrible disease" as an important reason for their poor diabetes control (although this factor ranked third in the patient survey), suggesting that the remaining $70 \%$ of the patients may have recognized the danger of diabetes. Thus, physicians and patients differed in their opinion in this regard. Given the physician's professional background in endocrinology, we would place more confidence in the physician's opinion. We believe that most of the patients did not have a sufficient understanding of diabetes, which may have impeded diabetes control; moreover, most of the patients may not have a strong interest in developing a thorough understanding of diabetes [18]. Therefore, we conducted a supplementary survey in a subgroup of 50 patients (Additional file 2). The questionnaire had two parts. Part 1contained basic information. Part 2 contained eight questions mainly about the diabetes complications (Question2-Question9). Patients got one score when 
they know one question in part 2. The results showed that among the 41 patients who rated themselves as someone with an understanding of the danger of diabetes, only $51.2 \%$ answered all of the questions correctly, and $66 \%$ answered $75 \%$ of the questions correctly. Although the patients who believed themselves to understand the danger of diabetes scored higher (mean score) than those who did not (6.78 vs $4.11, p=0.028$ ), they had a limited understanding of the acute and chronic complications of diabetes (Additional file 2). Thus, based on the measures suggested by the physicians during the survey, we recommend using more detailed educational materials to improve patient understanding of the danger of diabetes.

Moreover, we found that both physicians and patients (up to $40 \%$ ) considered self-monitoring of blood glucose as an important link of glucose control; specifically, "failure to monitor blood glucose regularly" ranked fourth in the physician survey and third in the patient survey as a factor for poor diabetes control. Economic conditions and (long-term) pain during testing were the two main reasons patients cited for failing to monitor their blood glucose regularly based on our clinical experience. The physicians suggested that health care coverage must first be expanded, particularly to cover test strips and needles used for glucose monitoring, similar to situations in some developing countries [11]. Prof. Weiping Jia (China) has also called on the Chinese government to expand health care coverage to cover the costs of glucose monitoring.

In addition, we found that false advertising and certain herbal medicines and health products were important potential barriers to optimal diabetes control in Chinese diabetic patients, suggesting that patients generally had a poor understanding of diabetes and urgently required truthful, accurate, and professional diabetes education. The physicians believed that regulating media campaigns and eliminating false advertising is one of the four public health measures necessary to improve the diabetes control rate. Herbal medicines and health products are unregulated in China, and the abuse of these products affected glucose control in $10 \%$ of the patients based on this survey. In addition, we did not include selfevaluation items in the physician survey, and therefore, we were unsure about the effect of iatrogenic events on patients' glucose control; however, the patient survey showed that iatrogenic events were not a major factor for uncontrolled glucose. Other factors, such as improving physician-patient communication, training of specialty nurses, and regular follow-ups, were considered important factors for improving glucose control.

The study design was well balanced. Based on the distribution of medical resources, we stratified and randomly sampled 18 representative hospitals of different levels in Shaanxi Province. However, this study had some limitations: Firstly, this study was conducted in only one province, and thus, the results may not be applicable to the whole of China. Secondly, the physician's professional skills were unknown, and thus, we were unable to evaluate the effect of physicians' professional skills on uncontrolled glucose. Thirdly, factor on economic reasons can be many, so the specific factor may not be available from the results. Fourthly, As the questionnaire only sought physician's perspective without patients' perspective for strategies of improvement, hence strategies as suggested by physicians may not be actually effective in improving patient's diabetes control. Lastly, we only included patients with type 2 diabetes, so the conclusion of this survey may not be generalized to other diabetic population. For example, accessing and maintaining contact with diabetes care services may be the most potential barrier to optimal diabetes control in young patients with type 1 diabetes [24].

\section{Conclusions}

This was the first formal large-scale multi-center survey of diabetes perception in physicians and patients in China. The survey revealed differences between the perspectives of physicians and patients on the potential barriers to optimal diabetes control, and the results showed that the main potential barriers to optimal diabetes control were patient's poor lifestyle interventions, limited understanding of the danger of diabetes, and poor self-monitoring of blood glucose. Thus, this study suggests that, as physicians and public health agencies, our primary focus about diabetes control in the future should still be put on diabetes education, particular the importance of lifestyle interventions and the danger of diabetes, by developing and promoting more educational materials. In addition, we hope that the Chinese government and society will expand health insurance coverage and take effective measures to regulate media campaigns.

\section{Additional files}

Additional file 1: Survey of the potential barriers to optimal diabetes control. (DOCX $30 \mathrm{~kb}$ )

Additional file 2: Survey of patients' perspectives for diabetes dangers. (DOCX $28 \mathrm{~kb})$

\section{Abbreviations \\ GDP: Gross Domestic Product; HbA1c: HemoglobinA1c; US: The United States of America}

\section{Acknowledgements}

We thank all of the physicians and participants of the study for their co-operation and generous participation. We also thank Prof. Yi Wan, Department of Health Statistics, School of Preventive Medicine, Fourth Military Medical University, Xi'an, China, for the critical reading of this manuscript. 


\section{Funding}

This study was partly supported by the Natural Science Foundation of Shaanxi Province, China (Grant No. 2013KTZB03-02-01). The funding bodies have not involved in the design of the study and collection, analysis, and interpretation of data and in writing the manuscript.

\section{Availability of data and materials}

The datasets used and/or analyzed during the current study are available from the corresponding author on reasonable request.

\section{Authors' contributions}

C.L., S.X. and J.M. contributed equally to the study. Q.J. and S.X. conceived and designed the study. C.L. and J.M. contributed to the data extraction, performed the analysis and interpreted the results. C.L. and S.X. wrote the first draft. A.J, Y.W, H.L, Y.J, M.S., Y.Z, Y.D, W.Y, X.L, S.S, H.T, G.J, GZ, L.W, M.Z, J.W, W.L, L.F, F.D, they all participated in the data collection and contributed to the revision of the final report. All authors read and approved the final manuscript.

\section{Ethics approval and consent to participate}

This study was approved by the Independent Ethics Committee of Xijing Hospital, the Fourth Military Medical University, China. Each patient and physician got informed consent and signed the consent form before the survey, while the physicians answered the questionnaire in an anonymous manner.

\section{Consent for publication}

Not applicable.

\section{Competing interests}

The authors declare that they have no competing interests.

\section{Publisher's Note}

Springer Nature remains neutral with regard to jurisdictional claims in published maps and institutional affiliations.

\section{Author details}

'Department of Endocrinology, Xijing Hospital, Air Force Medical University, 169 Changle Road West, Xi'an 710032, China. 'Department of Endocrinology, Yulin First Hospital, Yulin 719000, China. ${ }^{3}$ School of Nursing, Third Military Medical University, Chongqing 404100, China. ${ }^{4}$ Department of Endocrinology, Shanxi Provincial Peoples Hospital, Xi'an 710032, China. ${ }^{5}$ Department of Endocrinology, The Second Affiliated Hospital of Xi'an Jiaotong University, Xi'an 710032, China. 'Department of Endocrinology, The Fourth Hospital of Xi'an, Xi'an 710032, China. 'Department of Endocrinology, High-tech Hospital, Xi'an 710032, China. ${ }^{8}$ Department of Endocrinology, Changan Hospital, Xi'an 710032, China. ${ }^{9}$ Department of Endocrinology, Aerospace Hospital, Xi'an 710032, China. ${ }^{10}$ Department of Endocrinology, Huxian County People's Hospital, Xi'an 710032, China. ${ }^{11}$ Department of Endocrinology, Zhouzhi County People's Hospital, Xi'an 710032, China. ${ }^{12}$ Department of Endocrinology, The second people's Hospital of Shenmu county, Yulin 719000, China. ${ }^{13}$ Department of Endocrinology, Fugu County Hospital, Yulin 719000, China. ${ }^{14}$ Department of Endocrinology, Central Hospital of Baoji, Baoji 721000, China. ${ }^{15}$ Department of Endocrinology, Qishan County Hospital, Baoji 721000, China. ${ }^{16}$ Department of Endocrinology, Fengxiang County Hospital, Baoji 721000, China. ${ }^{17}$ Department of Endocrinology, Ankang Central Hospital, Ankang 725000, China.

${ }^{18}$ Department of Endocrinology, Xixiang County Hospital, Hanzhong 723000 , China. ${ }^{19}$ Department of Endocrinology, Xunyang County Hospital, Ankang 725000, China.

Received: 7 April 2018 Accepted: 30 November 2018 Published online: 12 December 2018

\section{References}

1. $X u Y$, Wang L, He J, et al. Prevalence and control of diabetes in Chinese adults. JAMA. 2013;310(9):948-59.

2. Wang $L$, Gao $P$, Zhang $M$, et al. Prevalence and ethnic pattern of diabetes and prediabetes in China in 2013. JAMA. 2017;317(24):2515-23.
3. Tiedt JA, Sloan RS. Perceived unsatisfactory care as a barrier to diabetes selfmanagement for Coeur d'Alene tribal members with type 2 diabetes. J Transcult Nurs. 2015;26(3):287-93.

4. Peyrot M, Rubin RR, Lauritzen T, et al. Psychosocial problems and barriers to improved diabetes management: results of the cross-National Diabetes Attitudes, wishes and needs (DAWN) study. Diabet Med. 2005;22:1379-85.

5. Schäfer I, Pawels $M$, Küver $C$, et al. Strategies for improving participation in diabetes education. A qualitative study. PLoS One. 2014;9(4):e95035.

6. Assaad-Khalil SH, Al Arouj M, Almaatouq M, et al. Barriers to the delivery of diabetes care in the Middle East and South Africa: a survey of 1,082 practising physicians in five countries. Int J Clin Pract. 2013;67(11):1144-50.

7. Abdoli S, Ashktorab T, Ahmadi F, et al. Religion, faith and the empowerment process: stories of Iranian people with diabetes. Int J Nurs Pract. 2011;17(3): 289-98.

8. Hayes RP, Fitzgerald JT, Jacober SJ. Primary care physician beliefs about insulin initiation in patients with type 2 diabetes. Int J Clin Pract. 2008;62(6): 860-8.

9. Agarwal G, Pierce M, Ridout D. The GP perspective: problems experienced in providing diabetes care in UK general practice. Diabet Med. 2002; 19(Suppl 4):13-20.

10. Holt RI, Nicolucci A, Kovacs Burns K, et al. Diabetes attitudes, wishes and needs second study (DAWN2 ${ }^{\mathrm{TM}}$ ): cross-national comparisons on barriers and resources for optimal care-healthcare professional perspective. Diabet Med. 2013;30(7):789-98.

11. Rätsep A, Oja I, Kalda R, Lember M. Family doctors' assessment of patientand health care system-related factors contributing to non-adherence to diabetes mellitus guidelines. Prim Care Diabetes. 2007;1(2):93-7.

12. Kaltman S, Talisman N, Serrano A, et al. Type 2 diabetes and depression: patient, family member, and primary care provider perspectives on the development of an integrated self-management intervention. Diabetes Educ. 2015;41(6):763-72.

13. van den Arend IJ, Rutten GE, Schrijvers GJ, Stolk RP. Experts' opinions on the profile of optimal care for patients with diabetes mellitus type 2 in the Netherlands. Neth J Med. 2001;58(6):225-31.

14. Strain $W D, \operatorname{Cos} X$, Hirst $M$, et al. Time to do more: addressing clinical inertia in the management of type 2 diabetes mellitus. Diabetes Res Clin Pract. 2014;105(3):302-12.

15. Suparee N, McGee P, Khan S, Pinyopasakul W. Life-long battle: perceptions of type 2 diabetes in Thailand. Chronic IIIn. 2015;11(1):56-68.

16. Torres HC, Rozemberg B, Amaral MA, Bodstein RC. Perceptions of primary healthcare professionals towards their role in type 2 diabetes mellitus patient education in Brazil. BMC Public Health. 2010;10:583.

17. Fransen MP, Beune EJ, Baim-Lance AM, et al. Diabetes self-management support for patients with low health literacy: perceptions of patients and providers. J Diabetes. 2015;7(3):418-25.

18. Lee V, McKay T, Ardern Cl. Awareness and perception of plant-based diets for the treatment and management of type 2 diabetes in a community education clinic: a pilot study. J Nutr Metab. 2015;2015:236234.

19. Gazmararian JA, Ziemer DC, Barnes C. Perception of barriers to self-care management among diabetic patients. Diabetes Educ. 2009;35(5):778-88.

20. Daoud N, Osman A, Hart TA, et al. Self-care management among patients with type 2 diabetes in East Jerusalem. Health Educ J. 2015;74(5):603-15.

21. Kamimura A, Christensen N, Myers K, et al. Health and diabetes self-efficacy: a study of diabetic and non-diabetic free clinic patients and family members. J Community Health. 2014;39(4):783-91.

22. Suraci C, Mulas F, Rossi MC, et al. Management of newly diagnosed patients with type 2 diabetes: what are the attitudes of physicians? A SUBITO!AMD survey on the early diabetes treatment in Italy. Acta Diabetol. 2012;49(6): 429-33.

23. Adams OP, Carter AO. Diabetes and hypertension guidelines and the primary health care practitioner in Barbados: knowledge, attitudes, practices and barriers--a focus group study. BMC Fam Pract. 2010;11:96.

24. Kibbey KJ, Speight J, Wong JL, et al. Diabetes care provision: barriers, enablers and service needs of young adults with type 1 diabetes from a region of social disadvantage. Diabet Med. 2013:30(7):878-84. 